\title{
1 Unaffected functional recovery after spinal cord contusions at different circadian
}

\section{2 times.}

4 Lukasz P. Slomnicki ${ }^{1,2}$, George Wei ${ }^{1,2,4,5}$, Darlene A. Burke ${ }^{1,2}$, Scott R. Whittemore ${ }^{1,2,3,4}$,

5 Sujata Saraswat Ohri ${ }^{1,2}$ and Michal Hetman ${ }^{1,2,3,4}$

$6 \quad{ }^{1}$ Kentucky Spinal Cord Injury Research Center and Departments of ${ }^{2}$ Neurological

7 Surgery, ${ }^{3}$ Anatomical Sciences \& Neurobiology, and ${ }^{4}$ Pharmacology \& Toxicology, and

$8{ }^{5} \mathrm{MD} / \mathrm{PhD}$ Program, University of Louisville School of Medicine, Louisville, KY, 40292

10 Corresponding author: Michal Hetman, M.D., Ph.D., Kentucky Spinal Cord Injury

11 Research Center, Departments of Neurological Surgery, Anatomical Sciences \&

12 Neurobiology, and Pharmacology \& Toxicology, 511 S. Floyd St., MDR616, University

13 of Louisville School of Medicine, Louisville, KY, 40292, Tel: (502) 852-3619 / Fax:

14 (502) 852-5148 / email: michal.hetman@1ouisville.edu

18 Keywords: spinal cord injury, circadian rhythm, time-of-day effects, locomotor

19 recovery, white matter, secondary injury 


\section{Abstract}

23 The circadian rhythms of gene expression drive diurnal oscillations of physiological

24 processes that determine the acute injury response including immunity, inflammation and

25 hemostasis. While outcomes of various acute injuries are affected by the time of day at

26 which the original insult occurred, such diurnal influences on recovery after spinal cord

27 injury (SCI) are unknown. We report that several key regulators of circadian gene

28 expression are differentially expressed in uninjured spinal cord tissue of naïve mice at

29 Zeitgeber time 1 (ZT1) or ZT12, where ZT0 or ZT12 are times when lights are turned on

30 or off, respectively. However, mice that received moderate, T9 contusive SCI at ZT0 or

31 ZT12 showed similar recovery of locomotion as determined using the ladder walking test

32 and the Basso mouse scale (BMS) over a 6 week post-injury period. Consistent with

33 those findings, terminal histological analysis revealed no significant differences in white

34 matter sparing at the injury epicenter. Therefore, locomotor recovery after thoracic

35 contusive SCI is not affected by the time of day at which the neurotrauma occurred at

36 least when comparing the beginning to the end of the mouse active period. 


\section{Introduction}

42 Time of day affects the incidence of various acute pathologies including

43 myocardial infarct (MI) and ischemic stroke [1]. Moreover, the severity of those injuries

44 may also be influenced by the time of day at which they occur $[2,3]$. Such effects stem

45 from circadian rhythmicity of biological processes that determine risk of a blood vessel

46 occlusion and/or rupture and/or modify the tissue injury response [1]. Thus, in morning

47 hours when the human active period begins, circadian maxima (acrophases) of blood

48 pressure, sympathetic tone, and hemostasis may explain higher occurrence and greater

49 severity of MI and stroke at that time [1]. In addition, circadian modulation of

50 metabolism, pro-inflammatory potential, immunity, anti-oxidant defenses and blood-

51 tissue barriers may directly affect sensitivity to acute injuries [1, 4-6].

53 Circadian rhythmicity of biological processes is mediated by oscillations of gene

54 expression produced by a set of conserved transcription factors (TFs) of the clock

55 pathway including BMAL1, CLOCK and NPAS2 [7]. The clock pathway in

56 hypothalamic suprachiasmatic nucleus $(\mathrm{SCN})$ neurons synchronizes clock pathways in

57 other cells of the body. The clock pathway TFs engage feedback loops that underlie

58 oscillating expression of them and their regulators.

In rodents, the activity of the effector outputs of the clock pathway in most non-

61 SCN tissues is low at ZT18-0 (late night/early morning) or high at ZT6-12

62 (afternoon/early evening), respectively [8] [9, 10]. Such oscillations coincide with

63 differential responses to such challenges as MI, infection, endotoxic shock or autoantigen 
64 exposure while genetic disruption of clock signaling nullifies those time of day effects

$65 \quad[11-15]$

66 Time of day effects are also documented in several models of acute brain injury

67 [16-21]. However, the maximum severity of brain damage peaked at distinctly different

68 times dependent on the model used [16-21]. Such variability suggests that unique, injury-

69 specific pathogenic mechanisms may be differentially sensitive to circadian regulation.

70 There are no reports of circadian effects on spinal cord injury (SCI).

BMAL1 is the principal non-redundant TF of the clock pathway output [7].

73 After moderate contusive SCI at the T9 level, Bmall ${ }^{-/}$mice showed enhanced locomotor

74 recovery, increased white matter sparing as well as reduced inflammation and improved

75 blood-spinal cord barrier function in the injury epicenter region [22]. Therefore, the

76 pathogenesis of SCI may be regulated by circadian rhythms. The current study was

77 initiated to test whether SCI outcomes differ if the injury occurs at the beginning or end

78 of the day, when the mouse active period ends or begins, respectively.

\section{Methods}

81 Animals. Six-week old C57Bl/6 wild-type female mice were obtained from the Jackson

82 Laboratory (Bar Harbor, ME). Animals were maintained in a 12:12 light-dark cycle (6:00

83 light on, 18:00 light off) with food and water available ad libitum for 2 weeks. After five

84 days of habituation to handling (performed in the same room where behavioral

85 assessments were later performed) mice were randomly assigned to

86 different experimental groups. All animal procedures were approved by the University of 
87 Louisville Institutional Animal Care and Use Committee and strictly adhered to NIH

88 guidelines.

89 Spinal cord injury. Avertin anesthesia, T9 spinal cord contusion (50 kdyn, IH impactor

90 /Infinite Horizons, Lexington, KY/) and post-surgery care were performed as previously

91 described [22, 23] (see Supplementary Methods for detailed information including

92 anesthesia and post-surgery analgesia). The surgeries were performed at ZT0-1.5 (6:00-

$937: 30, \mathrm{n}=11)$ or ZT12-13.5 $(18: 00-19: 30, \mathrm{n}=11)$ by the same team of investigators $12 \mathrm{~h}$

94 apart. Both groups were given identical post-surgical care and maintained under the same

95 conditions for six weeks. Three mice were lost (1 in ZT0 euthanized after accidental

96 rupture of the bladder during bladder expression, 2 in ZT12 were found dead at dpi 8 and

97 dpi 10). See Supplementary Table 1 for detailed contusion parameters (recorded force,

98 displacement, velocity).

99 Assessments of locomotor function. All behavioral assessments were performed at the

100 same time for both groups of mice by individuals without knowledge of group

101 assignment. Hindlimb locomotor function was evaluated in an open field using the Basso

102 Mouse Scale (BMS) by raters trained by Dr. Basso and colleagues at the Ohio State

103 University [24]. Evaluations were performed weekly, first before the injury to determine

104 baseline values, and then for six weeks starting at week 1 after SCI. The horizontal ladder

105 test was performed as described previously using Columbus Instruments Sensor and RS-

106232 Mini Counter (Columbus Instruments; Columbus, OH, USA, 2.5 mm rungs spaced

$1073.5 \mathrm{~cm}$ apart) [25]. Briefly, each animal underwent five stepping trials per session and the

108 total number of footfalls was quantified for the left and right limbs, respectively. A 
109 baseline session before SCI was followed by bi-weekly assessments starting at 2 weeks

110 post-injury.

111 White matter sparing was performed as described previously [23]. Briefly, after

112 completion of behavioral analyses (day post-injury 42), mice were deeply anaesthetized

113 and perfused transcardially with ice cold phosphate buffered saline (PBS) and then $4 \%$

114 paraformaldehyde (PFA) in PBS. Twenty $\mu \mathrm{m}$ serial transverse cryosections from a $4 \mathrm{~mm}$

115 spinal cord segment centered at the injury epicenter were stained for myelin with iron-

116 eriochrome cyanine (EC). For each animal, the section with least amount of myelinated

117 white matter was identified as the injury epicenter. White matter sparing was defined

118 as \% relative white matter area (per total section area) at the epicenter as compared to the

119 relative white matter area $2 \mathrm{~mm}$ rostral from the injury epicenter.

120 qRT-PCR analysis of circadian oscillations of gene expression. Naïve mice (coming from

121 the same batch of animals as that used for SCI and undergoing same handling

122 habituation) were deeply anesthetized and transcardially perfused with ice cold PBS at

123 ZT1 or ZT12 to collect a $5 \mathrm{~mm}$ segment of the thoracic spinal cord and the liver. Total

124 RNA extraction, synthesis of cDNA and SYBR Green-based qPCR analysis using the

$125 \Delta \Delta \mathrm{Ct}$ quantification method and Gapdh as a normalizer followed previously described

126 methodology [22]. See Supplementary Table 2 for primer information. The results were

127 compared to publicly available data on oscillations of the clock pathway mRNAs in

128 various tissues of 7-8 week old C57B16 mice including the brain stem, the cerebellum,

129 and the liver (http://circadb.hogeneschlab.org/mouse) [8].

130 Statistical analyses. Repeated measures ANOVA (RM ANOVA) followed by Tukey post

131 hoc tests was used for analyzing BMS and horizontal ladder locomotor recovery data. 
132 Gene expression and white matter sparing data were analyzed using the non-parametric

133 Mann-Whitney $u$-test.

\section{Results}

136 In various tissues including intact male or female rat spinal cord as well as male

137 C57B16 mouse brain stem, cerebellum or liver transcript levels for most clock pathway

138 mRNAs oscillate with a maximum amplitude at or around ZT0 and ZT12 [26] [8]

139 (http://circadb.hogeneschlab.org/mouse, Supplementary Fig. S1 and S2). Such

140 oscillations indicate activity of the clock pathway, as its core components are also clock

141 pathway-regulated at the transcriptional level [7]. Therefore, levels of selected clock

142 pathway transcripts were analyzed at ZT1 and ZT12 in the intact spinal cord and the liver

143 of naïve mice taken from the same cohort that was used for SCI studies. At ZT12, Bmal1

144 decreased by $42.5 \%$ in comparison to ZT1 (Fig. 1A). Consistent with increasing BMAL1

145 TF activity during the rodent inactive period, expression of several BMAL1 target genes

146 including Nr1d1, Nr1d2, Cry1, Per1, Per2, Per3, and Dbp was higher by 25-65\% at

147 ZT12. At that time, Bmal1 showed a 98\% decline in the liver with several of its target

148 genes showing strong increases by 50-90\% (Fig. 1B). These data validate natural

149 modulation of the clock pathway in the spinal cord and liver tissues of mice that were

150 used for SCI experiments. The findings are consistent with greater circadian regulation of

151 the transcriptome in the liver as compared to the CNS [8] and the reported maximum

152 amplitude for many clock pathway genes at the start and the end of the mouse active

153 period (Supplementary Fig. S1 and S2). 


\section{Figure 1. Circadian effects on expression of clock pathway mRNAs in the intact}

156 mouse spinal cord. Levels of mRNAs were determined at ZT1 and ZT12 by qPCR using

157 total RNA from the lower thoracic segment of the spinal cord (A) and the liver (B).

158 Gapdh was used as a normalizer for expression level determinations, $B 2 m$ was also

159 included as an additional normalizing transcript. Note that lower levels of Bmall

160 expression at ZT12 coincide with increased levels of several BMAL1 target genes

161 suggesting increased activity of the clock pathway output. The observed differences in

162 the spinal cord are consistent with reported maximal amplitudes of clock pathways

163 mRNA in other non-SCN regions of mouse brain at the beginning and the end of the

164 active period (Supplementary Fig. S1 and S2). For each transcript, data represent average

165 fold change of a time point with maximal expression $\pm \mathrm{SD} ;{ }^{*}, \mathrm{p}<0.05$, ns $>0.05$, $u$-test;

$166 \mathrm{n}=3$ mice/time point.

168 To determine whether oscillations of the clock pathway activity at the time of

169 injury correlate with a long-term locomotor recovery, moderate T9 SCI was performed at

170 ZT0 or ZT12. Mean displacement was similar for both groups (567.3 \pm 88.7 at ZT0 or

$171575.0 \pm 83.1 \mu \mathrm{m}$ at ZT12, $\mathrm{p}>0.05, t$-test) suggesting no difference in severity of the

172 primary injury. Similar recovery of hindlimb function was revealed with terminal BMS

173 scores of $4.90 \pm 0.61$ or $4.83 \pm 0.32$ and ladder errors of $9.64 \pm 3.04$ or $8.87 \pm 5.41$ for

174 ZT0 or ZT12, respectively (Fig. 2). In rodents with low thoracic level contusive SCI,

175 white matter loss at the injury epicenter is the primary determinant of functional deficits

$176[24,27]$. Therefore, the observed lack of altered functional recovery correlated well with 
177 the lack of significant differences in \% spared white matter between the groups (Fig. 2C,

178 D).

180 Figure 2. Similar locomotor recovery and white matter sparing after SCI at ZT0 or

181 ZT12. $\boldsymbol{A}, \boldsymbol{B}$, Locomotor recovery after T9 $50 \mathrm{kdyn}$ IH contusion was quantified using the

182 BMS (A) or the horizontal ladder test (B). The surgeries were done $12 \mathrm{~h}$ apart and all the 183 assessments were done at the same time. For either parameter, RM ANOVA showed

184 significant effects of time after injury but not time of injury (time of injury, BMS:

$185 \mathrm{~F}_{1,99}=0.64, \mathrm{p}=0.43$; time of injury, ladder: $\mathrm{F}_{1,46}=1.9, \mathrm{p}=0.17$; see Supplementary Table 3

186 for more results). $\boldsymbol{C}, \boldsymbol{D}$, WMS was analyzed after completion of behavioral assessments 6

187 weeks after SCI. $\boldsymbol{C}$, Representative images of EC myelin staining in spinal cord sections

188 cut through the injury epicenter. $\boldsymbol{D}$, similar \% spared white matter in ZT0 SCI and ZT12

189 SCI mice (ns, $\mathrm{p}>0.05, u$-test). Data represent means $\pm \mathrm{SD}$.

\section{Discussion}

192 Our results suggest that after contusive SCI, impairment of hindlimb function and

193 its subsequent recovery is similar irrespective of whether the injury occurred at the

194 beginning or the end of the mouse active period. In addition, consistent with published

195 clock pathway gene expression studies of rodent CNS tissue, we report relatively higher

196 or lower activity of the clock pathway at those two times of day, respectively $[8,9]$. As

197 reliable determination of the nadir and the zenith for a circadian-regulated mRNA

198 requires probing at a minimum of 6 different timepoints/day across at least two days, we

199 do not have sufficient data to determine the spinal cord rhythm of clock pathway 
200 transcripts [28]. However, we can rely on published reports and/or publicly available data

201 sets from other areas of the mouse CNS as well as other organs that, apart from SCN,

202 show similar phase of oscillations for all major mediators of the clock pathway with a

203 zenith of the clock pathway activity by the start of the active period and a nadir at its end

204 (Supplementary Fig. S1 and S2). In addition, our spinal cord or liver data show that clock

205 pathway mRNA changes at ZT1 and ZT12 are of similar magnitude as maximal

206 amplitudes reported in the mouse CNS or liver, respectively (Fig 1, Supplementary Fig.

$207 \mathrm{~S} 1$ and S2). One notable exception is $\mathrm{Nr} 1 d 1 / \mathrm{Nr} 1 \mathrm{~d} 2$ which peaks in the middle of the

208 inactive period (Supplementary Fig. S1). Therefore, our analysis likely underestimates its

209 maximal circadian oscillations in the spinal cord and the liver. However, the presented

210 findings confirm that in the cohort of mice that was used for SCI studies, natural

211 circadian oscillations of gene expression occurred in the spinal cord and the liver and that

212 their phase was likely similar to that reported in rodents. Thus, circadian rhythmicity of

213 gene expression and its effector biological processes could be considered as a potential

214 variable that determines outcome of SCI.

216 While this report is the first to address the question of time of day effects on

217 pathogenesis of SCI, others have documented existence of circadian modulation in

218 several acute CNS injury models. Thus, in a middle cerebral artery occlusion (MCAO)

219 model of rat stroke, acute infarct volume was three times larger with a stroke at ZT22

220 than at ZT10 [16]. However, relatively moderate circadian effects were reported in a

221 murine MCAO model [21]. Global brain ischemia at ZT14 resulted in maximal

222 hippocampal apoptosis in a rat, while the greatest hippocampal damage followed ZT6 
223 ischemia in mice $[17,19]$. In a mouse subarachnoid hemorrhage model, less apoptosis

224 was observed in the hippocampus and the cortex when the insults occurred at ZT12 as

225 compared to ZT2 [20]. Closed skull traumatic brain injury (TBI) in rats at ZT17 reduced

226 brain damage area and acute mortality with a transient improvement in locomotor

227 function as compared to ZT5 TBI [18]. These results indicate that dependent on the CNS

228 injury model used, injuries that occurred in the second half of the active phase through

229 the start of the inactive phase, when clock pathway activity reaches a nadir, produced

230 maximal or minimal pathology at acute/subacute phases. While the current SCI time of

231 day study did not reveal significant differences in long term recovery or tissue sparing,

232 the aforementioned reports of circadian effects focused on acute/subacute pathological

233 changes with long term functional recovery or lesion healing either not examined or

234 unaffected. Hence, in several types of acute CNS injury in rodents, including mouse

235 moderate SCI, the time of insult may have, at most, only transient effects on secondary

236 damage without long lasting functional impact.

238 One could argue that the severity of the SCI paradigm used for this work may

239 have been too high to detect time of injury effects. This is unlikely as this moderate IH

240 contusion has been used in several mouse studies in which locomotor recovery and

241 terminal white matter sparing were increased or decreased by various genetic or

242 pharmacological manipulations that targeted the secondary injury cascades $[22,23,29$ -

243 32]. Hence, excessive primary damage is unlikely to explain our negative findings. 
Why is post-SCI recovery not affected by the time of injury despite our prior

246 findings that $\mathrm{Bmal}^{-/-}$mice have improved outcome [22]? While there are many possible

247 explanations to reconcile those observations, one should note that BMAL1 has been

248 implicated in gene expression regulation beyond circadian rhythms [33, 34]. Loss of such

249 a regulatory activity was proposed to contribute to a neurological phenotype of BMAL1-

250 deficient mice and may have also played a role in SCI outcome [33]. Moreover, altered

251 clock pathway/BMAL1 activity after, but not before, and/or at the time of SCI may be a

252 critical contributor to secondary injury and long-term recovery after SCI. Interestingly,

253 acute increases of at least some clock pathway components were observed in the injured

254 mouse spinal cord tissue [22]. Those findings may indicate that after SCI, the clock

255 pathway is reset to a new, post-injury time. Hence, modulation of the secondary damage

256 by the injury-regulated activity of the clock pathway may override any earlier influences

257 from natural circadian oscillations. Lastly, a confounding factor that may alter the

258 significance of natural clock pathway oscillations in experimental SCI may be pre- and

259 post-surgery care including such potential clock-resetting stimuli as anesthesia and

260 analgesia $[35,36]$.

\section{Conclusions}

263 Current work using moderate contusive SCI indicates no major injury time of day

264 effects on long term locomotor recovery and white matter integrity in mice at least when

265 comparing the beginning to the end of the mouse active period. Obtaining clinical data to

266 test if time of injury affects SCI outcome in humans will be extremely difficult as

267 confounding injury co-morbidities and variable extents of spontaneous recovery will 
268 restrict necessary samples sizes $[37,38]$. However, future preclinical analyses to address

269 potential effects on additional outcomes such as immune system dysregulation [39] may

270 provide insight into potential novel therapeutic avenues that could be acutely initiated

271 depending on time of injury.

272

273 Acknowledgements. This work was supported by Kentucky Spinal Cord and Head

274 Injury Research Trust (contract\# 18-2), NS108529-01, NS114404, Norton Healthcare,

275 and the Commonwealth of Kentucky Challenge for Excellence. We thank Christine

276 Yarberry for surgical assistance, Johnny Morehouse and Jason Beare for behavioral

277 analyses.

278

279

280

281 


\section{References}

284 1. Durgan DJ, Young ME. The cardiomyocyte circadian clock: emerging roles in

285 health and disease. Circ Res. 2010;106(4):647-58. Epub 2010/03/06. doi:

286 10.1161/CIRCRESAHA.109.209957. PubMed PMID: 20203314; PubMed Central

287 PMCID: PMCPMC3223121.

288 2. Traverse JH. Of mice and men: the quest to determine a circadian basis for

289 myocardial protection in ischemia/reperfusion injury. Circ Res. 2013;112(10):e115-7.

290 Epub 2013/05/11. doi: 10.1161/CIRCRESAHA.113.301079. PubMed PMID: 23661714.

291 3. Shokri HM, El Nahas NM, Aref HM, Dawood NL, Abushady EM, Abd Eldayem

292 EH, et al. Factors related to time of stroke onset versus time of hospital arrival: A SITS

293 registry-based study in an Egyptian stroke center. PLoS One. 2020;15(9):e0238305. Epub

294 2020/09/12. doi: 10.1371/journal.pone.0238305. PubMed PMID: 32915811; PubMed

295 Central PMCID: PMCPMC7485782.

296 4. Musiek ES, Holtzman DM. Mechanisms linking circadian clocks, sleep, and

297 neurodegeneration. Science. 2016;354(6315):1004-8. Epub 2016/11/26. doi: tzman

298 PubMed PMID: 27885006; PubMed Central PMCID: PMC5219881.

299 5. Carroll RG, Timmons GA, Cervantes-Silva MP, Kennedy OD, Curtis AM.

300 Immunometabolism around the Clock. Trends in molecular medicine. 2019;25(7):612-25.

301 Epub 2019/06/04. doi: 10.1016/j.molmed.2019.04.013. PubMed PMID: 31153819.

302 6. Cuddapah VA, Zhang SL, Sehgal A. Regulation of the Blood-Brain Barrier by

303 Circadian Rhythms and Sleep. Trends Neurosci. 2019;42(7):500-10. Epub 2019/06/30. 
304 doi: 10.1016/j.tins.2019.05.001. PubMed PMID: 31253251; PubMed Central PMCID:

305 PMCPMC6602072.

$3067 . \quad$ Lowrey PL, Takahashi JS. Genetics of circadian rhythms in Mammalian model

307 organisms. Advances in genetics. 2011;74:175-230. Epub 2011/09/20. doi:

308 10.1016/B978-0-12-387690-4.00006-4. PubMed PMID: 21924978; PubMed Central

309 PMCID: PMC3709251.

310 8. Zhang R, Lahens NF, Ballance HI, Hughes ME, Hogenesch JB. A circadian gene

311 expression atlas in mammals: implications for biology and medicine. Proceedings of the

312 National Academy of Sciences of the United States of America. 2014;111(45):16219-24.

313 Epub 2014/10/29. doi: 10.1073/pnas.1408886111. PubMed PMID: 25349387; PubMed

314 Central PMCID: PMC4234565.

3159 9. Hor CN, Yeung J, Jan M, Emmenegger Y, Hubbard J, Xenarios I, et al. Sleep-

316 wake-driven and circadian contributions to daily rhythms in gene expression and

317 chromatin accessibility in the murine cortex. Proceedings of the National Academy of

318 Sciences of the United States of America. 2019;116(51):25773-83. Epub 2019/11/30. doi:

319 10.1073/pnas.1910590116. PubMed PMID: 31776259; PubMed Central PMCID:

320 PMCPMC6925978.

321 10. Yang N, Smyllie NJ, Morris H, Goncalves CF, Dudek M, Pathiranage DRJ, et al.

322 Quantitative live imaging of Venus::BMAL1 in a mouse model reveals complex

323 dynamics of the master circadian clock regulator. PLoS Genet. 2020;16(4):e1008729.

324 Epub 2020/05/01. doi: 10.1371/journal.pgen.1008729. PubMed PMID: 32352975;

325 PubMed Central PMCID: PMCPMC7217492. 
326 11. Durgan DJ, Pulinilkunnil T, Villegas-Montoya C, Garvey ME, Frangogiannis NG,

327 Michael LH, et al. Short communication: ischemia/reperfusion tolerance is time-of-day-

328 dependent: mediation by the cardiomyocyte circadian clock. Circ Res. 2010;106(3):546-

329 50. Epub 2009/12/17. doi: 10.1161/CIRCRESAHA.109.209346. PubMed PMID:

330 20007913; PubMed Central PMCID: PMCPMC3021132.

331 12. Nguyen KD, Fentress SJ, Qiu Y, Yun K, Cox JS, Chawla A. Circadian gene

332 Bmall regulates diurnal oscillations of Ly6C(hi) inflammatory monocytes. Science.

333 2013;341(6153):1483-8. Epub 2013/08/24. doi: 10.1126/science.1240636. PubMed

334 PMID: 23970558; PubMed Central PMCID: PMCPMC3836670.

335 13. Deng W, Zhu S, Zeng L, Liu J, Kang R, Yang M, et al. The Circadian Clock

336 Controls Immune Checkpoint Pathway in Sepsis. Cell reports. 2018;24(2):366-78. Epub

337 2018/07/12. doi: 10.1016/j.celrep.2018.06.026. PubMed PMID: 29996098; PubMed

338 Central PMCID: PMCPMC6094382.

339 14. Curtis AM, Fagundes CT, Yang G, Palsson-McDermott EM, Wochal P,

340 McGettrick AF, et al. Circadian control of innate immunity in macrophages by miR-155

341 targeting Bmal1. Proceedings of the National Academy of Sciences of the United States

342 of America. 2015;112(23):7231-6. Epub 2015/05/23. doi: 10.1073/pnas.1501327112.

343 PubMed PMID: 25995365; PubMed Central PMCID: PMCPMC4466714.

344 15. Sutton CE, Finlay CM, Raverdeau M, Early JO, DeCourcey J, Zaslona Z, et al.

345 Loss of the molecular clock in myeloid cells exacerbates T cell-mediated CNS

346 autoimmune disease. Nature communications. 2017;8(1):1923. Epub 2017/12/14. doi:

347 10.1038/s41467-017-02111-0. PubMed PMID: 29234010; PubMed Central PMCID:

348 PMCPMC5727202. 
349 16. Vinall PE, Kramer MS, Heinel LA, Rosenwasser RH. Temporal changes in

350 sensitivity of rats to cerebral ischemic insult. J Neurosurg. 2000;93(1):82-9. Epub

351 2000/07/07. doi: 10.3171/jns.2000.93.1.0082. PubMed PMID: 10883909.

352 17. Tischkau SA, Cohen JA, Stark JT, Gross DR, Bottum KM. Time-of-day affects

353 expression of hippocampal markers for ischemic damage induced by global ischemia.

354 Experimental neurology. 2007;208(2):314-22. Epub 2007/10/16. doi:

355 10.1016/j.expneurol.2007.09.003. PubMed PMID: 17936274.

356 18. Martinez-Vargas M, Gonzalez-Rivera R, Soto-Nunez M, Cisneros-Martinez M,

357 Huerta-Saquero A, Morales-Gomez J, et al. Recovery after a traumatic brain injury

358 depends on diurnal variations effect of cystatin C. Neurosci Lett. 2006;400(1-2):21-4.

359 Epub 2006/03/08. doi: 10.1016/j.neulet.2006.02.010. PubMed PMID: 16519999.

360 19. Weil ZM, Karelina K, Su AJ, Barker JM, Norman GJ, Zhang N, et al. Time-of-

361 day determines neuronal damage and mortality after cardiac arrest. Neurobiol Dis.

362 2009;36(2):352-60. Epub 2009/08/12. doi: 10.1016/j.nbd.2009.07.032. PubMed PMID:

363 19664712; PubMed Central PMCID: PMCPMC2760634.

364 20. Schallner N, Lieberum JL, Gallo D, LeBlanc RH, 3rd, Fuller PM, Hanafy KA, et

365 al. Carbon Monoxide Preserves Circadian Rhythm to Reduce the Severity of

366 Subarachnoid Hemorrhage in Mice. Stroke. 2017;48(9):2565-73. Epub 2017/07/28. doi:

367 10.1161/STROKEAHA.116.016165. PubMed PMID: 28747460; PubMed Central

368 PMCID: PMCPMC5575974.

369 21. Beker MC, Caglayan B, Yalcin E, Caglayan AB, Turkseven S, Gurel B, et al.

370 Time-of-Day Dependent Neuronal Injury After Ischemic Stroke: Implication of Circadian

371 Clock Transcriptional Factor Bmal1 and Survival Kinase AKT. Mol Neurobiol. 
372 2018;55(3):2565-76. Epub 2017/04/20. doi: 10.1007/s12035-017-0524-4. PubMed

373 PMID: 28421530.

374 22. Slomnicki LP, Myers SA, Saraswat Ohri S, Parsh MV, Andres KR, Chariker JH,

375 et al. Improved locomotor recovery after contusive spinal cord injury in Bmal1(-/-) mice

376 is associated with protection of the blood spinal cord barrier. Scientific reports.

377 2020;10(1):14212. Epub 2020/08/28. doi: 10.1038/s41598-020-71131-6. PubMed PMID:

378 32848194; PubMed Central PMCID: PMCPMC7450087.

379 23. Ohri SS, Maddie MA, Zhao Y, Qiu MS, Hetman M, Whittemore SR. Attenuating

380 the endoplasmic reticulum stress response improves functional recovery after spinal cord

381 injury. Glia. 2011;59(10):1489-502. Epub 2011/06/04. doi: 10.1002/glia.21191. PubMed

382 PMID: 21638341; PubMed Central PMCID: PMC3391751.

383 24. Basso DM, Fisher LC, Anderson AJ, Jakeman LB, McTigue DM, Popovich PG.

384 Basso Mouse Scale for locomotion detects differences in recovery after spinal cord injury

385 in five common mouse strains. J Neurotrauma. 2006;23(5):635-59. Epub 2006/05/13. doi:

386 10.1089/neu.2006.23.635. PubMed PMID: 16689667.

387 25. Kim JH, Song SK, Burke DA, Magnuson DS. Comprehensive locomotor

388 outcomes correlate to hyperacute diffusion tensor measures after spinal cord injury in the

389 adult rat. Experimental neurology. 2012;235(1):188-96. Epub 2011/11/29. doi:

390 10.1016/j.expneurol.2011.11.015. PubMed PMID: 22119625; PubMed Central PMCID:

$391 \quad$ PMC3334428.

392 26. Gaudet AD, Fonken LK, Ayala MT, Bateman EM, Schleicher WE, Smith EJ, et

393 al. Spinal Cord Injury in Rats Disrupts the Circadian System. eNeuro. 2018;5(6). Epub 
394 2019/01/11. doi: 10.1523/ENEURO.0328-18.2018. PubMed PMID: 30627655; PubMed

395 Central PMCID: PMCPMC6325559.

396 27. Magnuson DS, Trinder TC, Zhang YP, Burke D, Morassutti DJ, Shields CB.

397 Comparing deficits following excitotoxic and contusion injuries in the thoracic and

398 lumbar spinal cord of the adult rat. Experimental neurology. 1999;156(1):191-204. Epub

399 1999/04/08. doi: 10.1006/exnr.1999.7016. PubMed PMID: 10192790.

400 28. Hughes ME, Abruzzi KC, Allada R, Anafi R, Arpat AB, Asher G, et al.

401 Guidelines for Genome-Scale Analysis of Biological Rhythms. J Biol Rhythms.

402 2017;32(5):380-93. Epub 2017/11/04. doi: 10.1177/0748730417728663. PubMed PMID:

403 29098954; PubMed Central PMCID: PMCPMC5692188.

404 29. Ohri SS, Hetman M, Whittemore SR. Restoring endoplasmic reticulum

405 homeostasis improves functional recovery after spinal cord injury. Neurobiol Dis.

406 2013;58:29-37. Epub 2013/05/11. doi: 10.1016/j.nbd.2013.04.021. PubMed PMID:

407 23659896; PubMed Central PMCID: PMC3748169.

408 30. Myers SA, Andres KR, Hagg T, Whittemore SR. CD36 deletion improves

409 recovery from spinal cord injury. Experimental neurology. 2014;256:25-38. Epub

410 2014/04/03. doi: 10.1016/j.expneurol.2014.03.016. PubMed PMID: 24690303; PubMed

411 Central PMCID: PMC4086463.

412 31. Saraswat Ohri S, Bankston AN, Mullins SA, Liu Y, Andres KR, Beare JE, et al.

413 Blocking Autophagy in Oligodendrocytes Limits Functional Recovery after Spinal Cord

414 Injury. J Neurosci. 2018;38(26):5900-12. Epub 2018/05/26. doi:

415 10.1523/JNEUROSCI.0679-17.2018. PubMed PMID: 29793971; PubMed Central

416 PMCID: PMC6021994. 
417 32. Saraswat Ohri S, Burke DA, Andres KR, Hetman M, Whittemore SR. Acute

418 Neural and Proteostasis Messenger Ribonucleic Acid Levels Predict Chronic Locomotor

419 Recovery after Contusive Spinal Cord Injury. J Neurotrauma. 2020. Epub 2020/10/21.

420 doi: 10.1089/neu.2020.7258. PubMed PMID: 33076743.

421 33. Musiek ES, Lim MM, Yang G, Bauer AQ, Qi L, Lee Y, et al. Circadian clock

422 proteins regulate neuronal redox homeostasis and neurodegeneration. J Clin Invest.

423 2013;123(12):5389-400. Epub 2013/11/26. doi: 10.1172/JCI70317. PubMed PMID:

424 24270424; PubMed Central PMCID: PMC3859381.

425 34. Yang G, Chen L, Grant GR, Paschos G, Song WL, Musiek ES, et al. Timing of

426 expression of the core clock gene Bmall influences its effects on aging and survival.

427 Science translational medicine. 2016;8(324):324ra16. Epub 2016/02/05. doi:

428 10.1126/scitranslmed.aad3305. PubMed PMID: 26843191; PubMed Central PMCID:

429 PMC4870001.

430 35. Imai R, Makino H, Katoh T, Kimura T, Kurita T, Hokamura K, et al. Desflurane

431 anesthesia shifts the circadian rhythm phase depending on the time of day of anesthesia.

432 Scientific reports. 2020;10(1):18273. Epub 2020/10/28. doi: 10.1038/s41598-020-75434-

433 6. PubMed PMID: 33106509; PubMed Central PMCID: PMCPMC7588451.

434 36. Jirkof P, Tourvieille A, Cinelli P, Arras M. Buprenorphine for pain relief in mice:

435 repeated injections vs sustained-release depot formulation. Lab Anim. 2015;49(3):177-

436 87. Epub 2014/12/10. doi: 10.1177/0023677214562849. PubMed PMID: 25488320.

437 37. Geisler FH, Coleman WP, Grieco G, Poonian D, Sygen Study G. Measurements

438 and recovery patterns in a multicenter study of acute spinal cord injury. Spine. 
439 2001;26(24 Suppl):S68-86. Epub 2002/01/24. doi: 10.1097/00007632-200112151-00014.

440 PubMed PMID: 11805613.

441 38. Steeves JD, Lammertse D, Curt A, Fawcett JW, Tuszynski MH, Ditunno JF, et al.

442 Guidelines for the conduct of clinical trials for spinal cord injury (SCI) as developed by

443 the ICCP panel: clinical trial outcome measures. Spinal cord. 2007;45(3):206-21. Epub

444 2006/12/21. doi: 10.1038/sj.sc.3102008. PubMed PMID: 17179972.

445 39. Zhang Y, Guan Z, Reader B, Shawler T, Mandrekar-Colucci S, Huang K, et al.

446 Autonomic dysreflexia causes chronic immune suppression after spinal cord injury. J

447 Neurosci. 2013;33(32):12970-81. Epub 2013/08/09. doi: 10.1523/JNEUROSCI.1974-

448 13.2013. PubMed PMID: 23926252; PubMed Central PMCID: PMC3735880. 
453 Figure 1. Circadian effects on expression of clock pathway mRNAs in the intact

454 mouse spinal cord. Levels of mRNAs were determined at ZT1 and ZT12 by qPCR using 455 total RNA from the lower thoracic segment of the spinal cord (A) and the liver (B).

456 Gapdh was used as a normalizer for expression level determinations, $B 2 m$ was also

457 included as an additional normalizing transcript. Note that lower levels of Bmall

458 expression at ZT12 coincide with increased levels of several BMAL1 target genes

459 suggesting increased activity of the clock pathway output. For each transcript, data

460 represent average fold change of a time point with maximal expression $\pm \mathrm{SD} ; *, \mathrm{p}<0.05$, $461 \mathrm{~ns}>0.05, u$-test; $\mathrm{n}=3$ mice/time point.

463 Figure 2. Similar locomotor recovery and white matter sparing after SCI at ZT0 or

464 ZT12. $\boldsymbol{A}, \boldsymbol{B}$, Locomotor recovery after T9 $50 \mathrm{kdyn}$ IH contusion was quantified using the

465 BMS (A) or the horizontal ladder test (B). The surgeries were done $12 \mathrm{~h}$ apart and all the

466 assessments were done at the same time. For either parameter, RM ANOVA showed

467 significant effects of time after injury but not time of injury (time of injury, BMS:

$468 \mathrm{~F}_{1,99}=0.64, \mathrm{p}=0.43$; time of injury, ladder: $\mathrm{F}_{1,46}=1.9, \mathrm{p}=0.17$; see Supplementary Table 3

469 for more results). $\boldsymbol{C}, \boldsymbol{D}$, WMS was analyzed after completion of behavioral assessments 6

470 weeks after SCI. $\boldsymbol{C}$, Representative images of EC myelin staining in spinal cord sections

471 cut through the injury epicenter. $\boldsymbol{D}$, similar \% spared white matter in ZT0 SCI and ZT12

472 SCI mice (ns, $\mathrm{p}>0.05, u$-test). Data represent means $\pm \mathrm{SD}$. 


\section{Supplementary Information}

477 Supplementary Methods. Spinal cord injury and post-surgery animal care.

479 Supplementary Table S1. Contusion parameters for each individual animal

481 Supplementary Table S2. qPCR primers

482

483 Supplementary Table S3. Results of RM ANOVA for BMS and ladder test

485 Supplementary Figures S1 and S2. Circadian oscillations of selected clock pathway

486 mRNAs in the brain stem, the cerebellum and the liver of C57Bl6 mice. The data are

487 from the publicly available circadian transcriptome database

488 (http://circadb.hogeneschlab.org/mouse). All presented mRNAs show significant

489 circadian oscillations in all three tissues (JTK p <0.05) except Cryl (non-significant

490 cycling in the brain stem $/ \mathrm{JTK} \mathrm{p}=0.058 /$ ) and $B 2 m$ (no cycling in any tissue $/ \mathrm{JTK} \mathrm{p}=1 /$ ). 
A

B

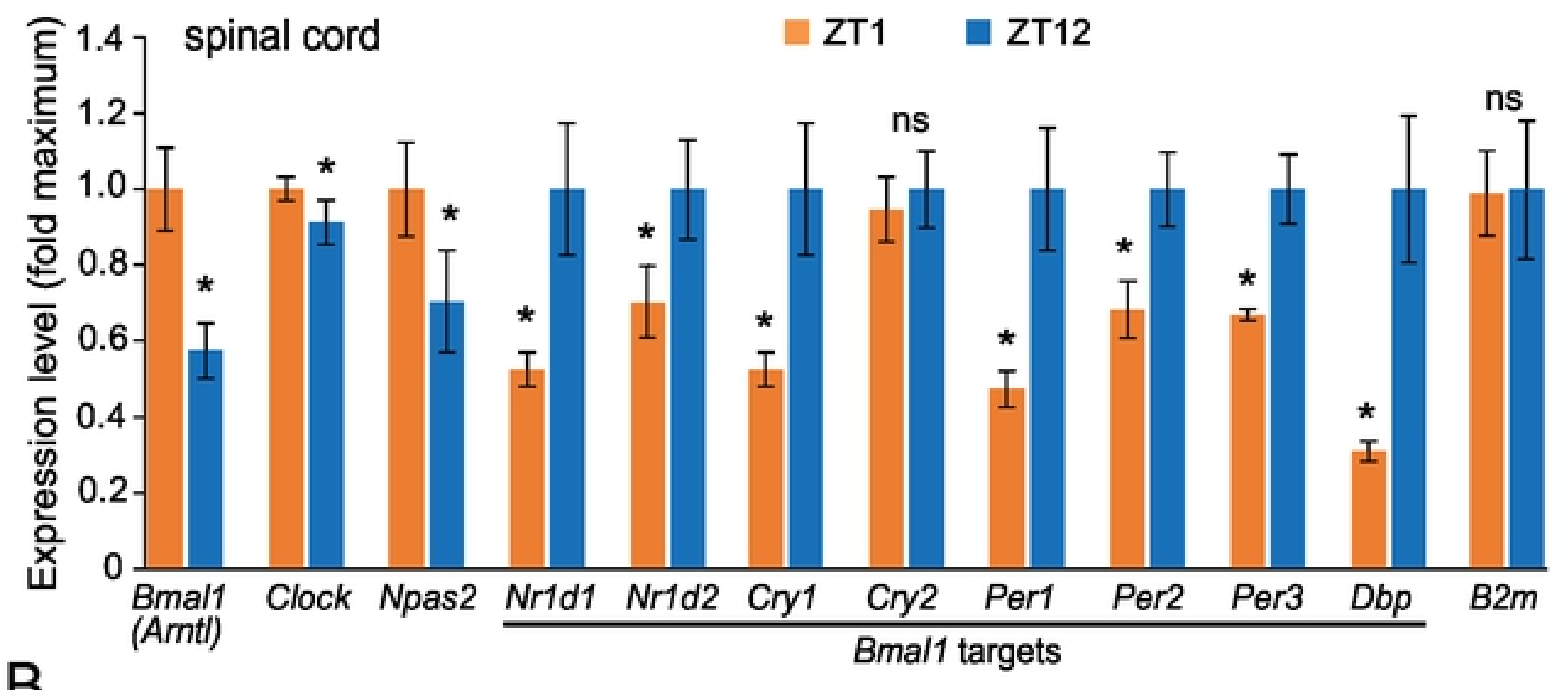
bioRxiv preprint doi: https://doi.org/10.1101/2021.03.30.437713; this version posted March 30, 2021. The copyright holder for this preprint
(which was not certified by peer review) is the author/funder, who has granted bioRxiv a license to display the preprint in perpetuity. It is made

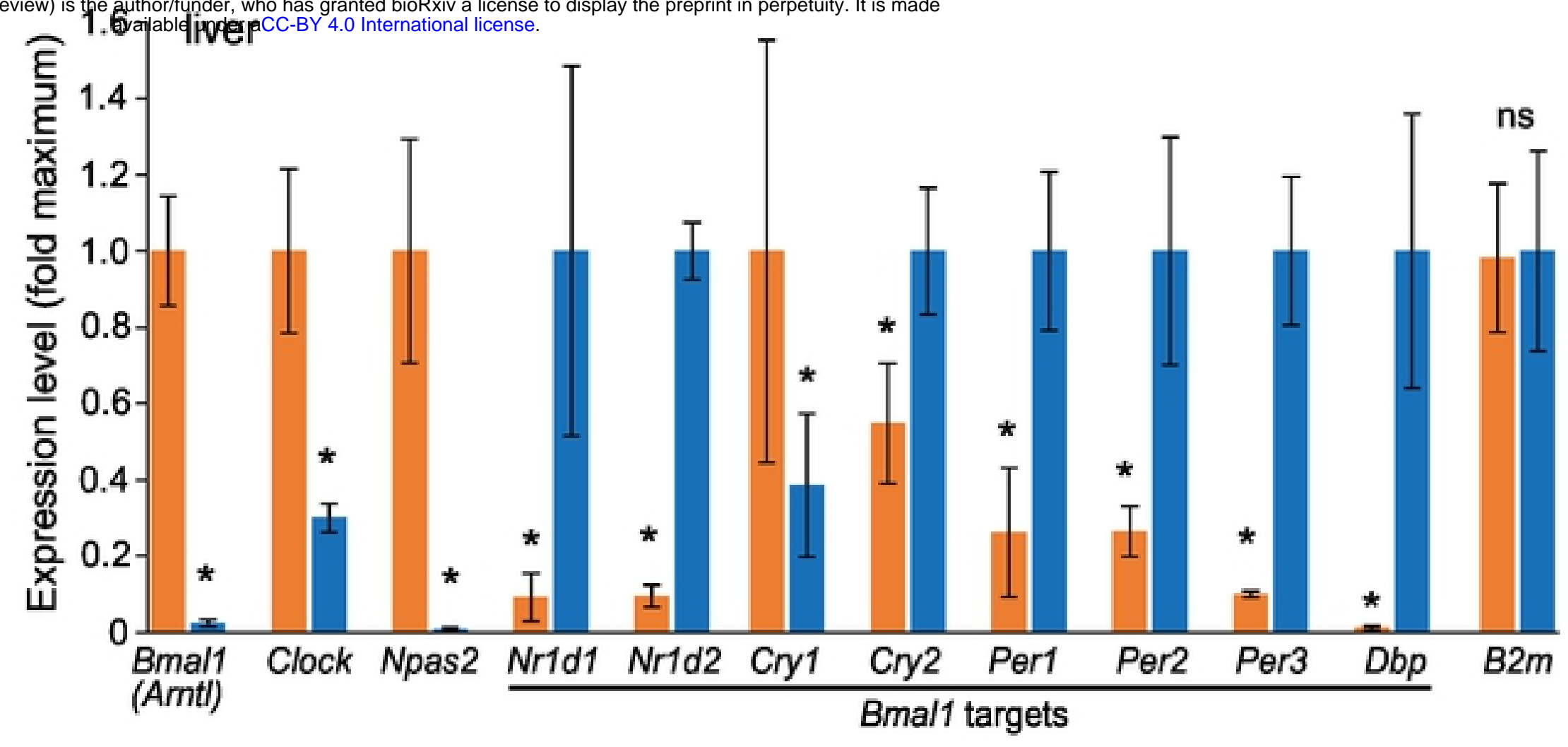

Slomnicki et al., Figure 1

Fig. 1 
A

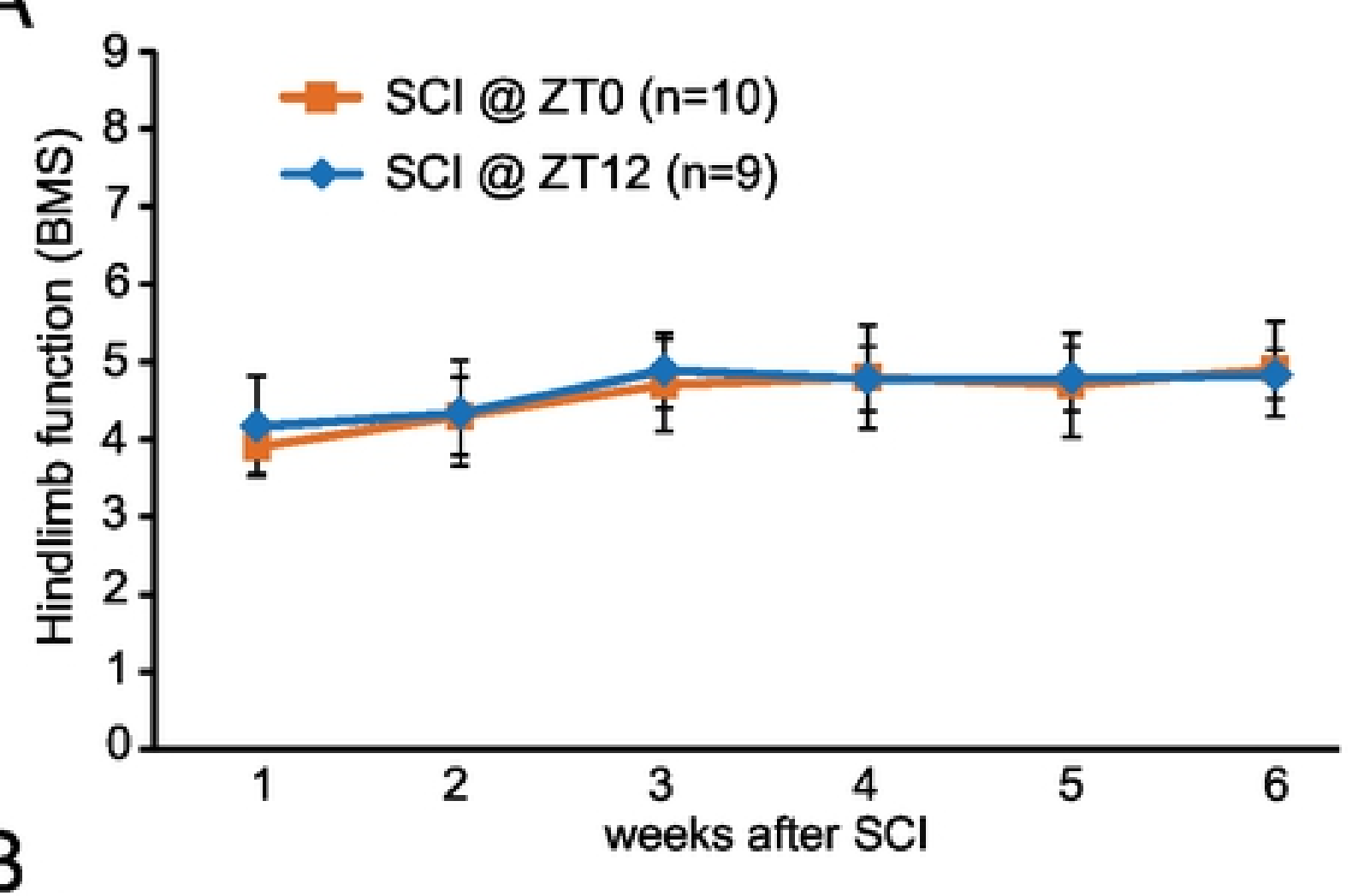

B

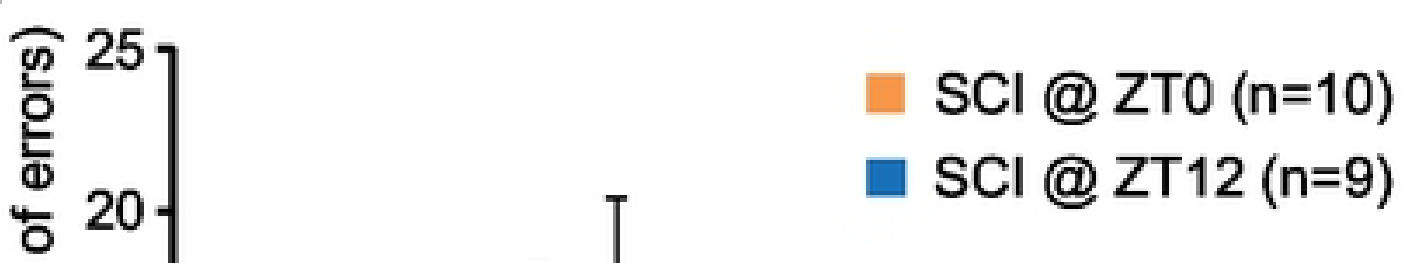

bioRxiv preprint doi: https://doi.org/10 1101/2021

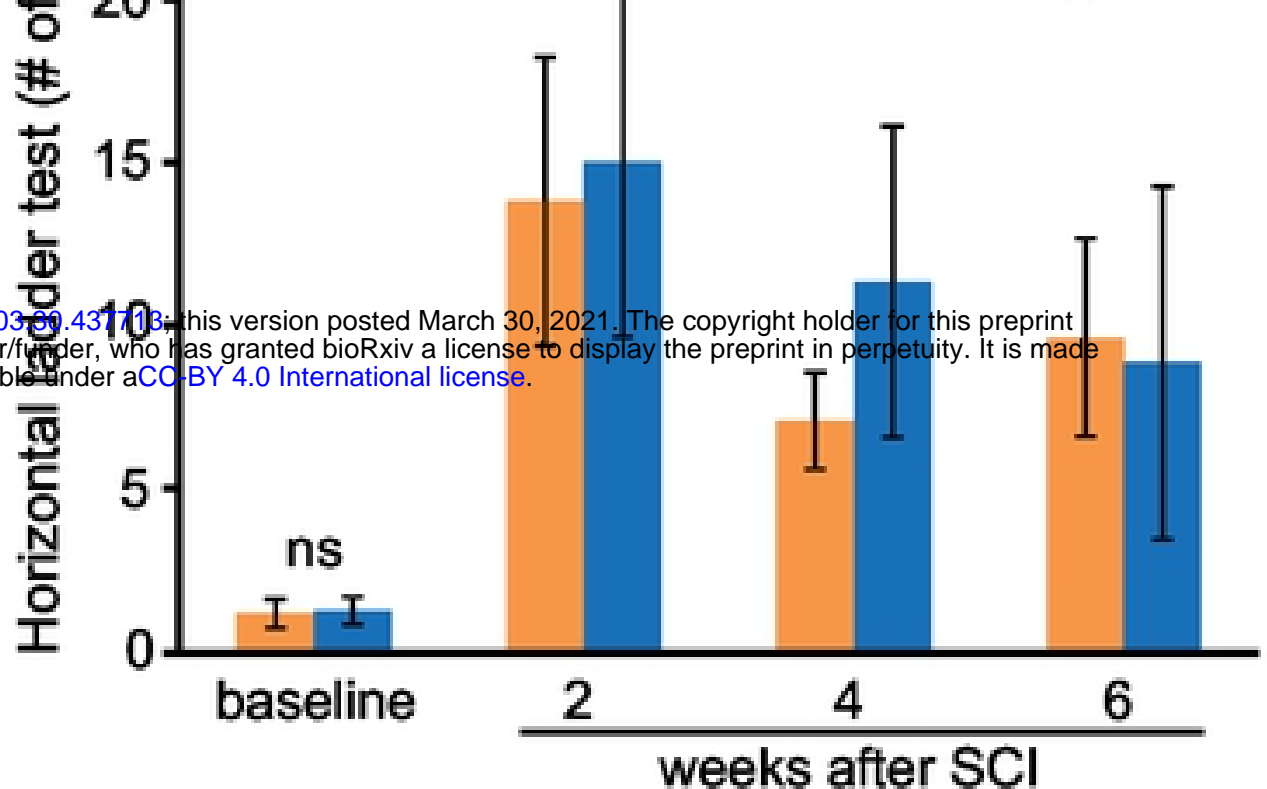

C

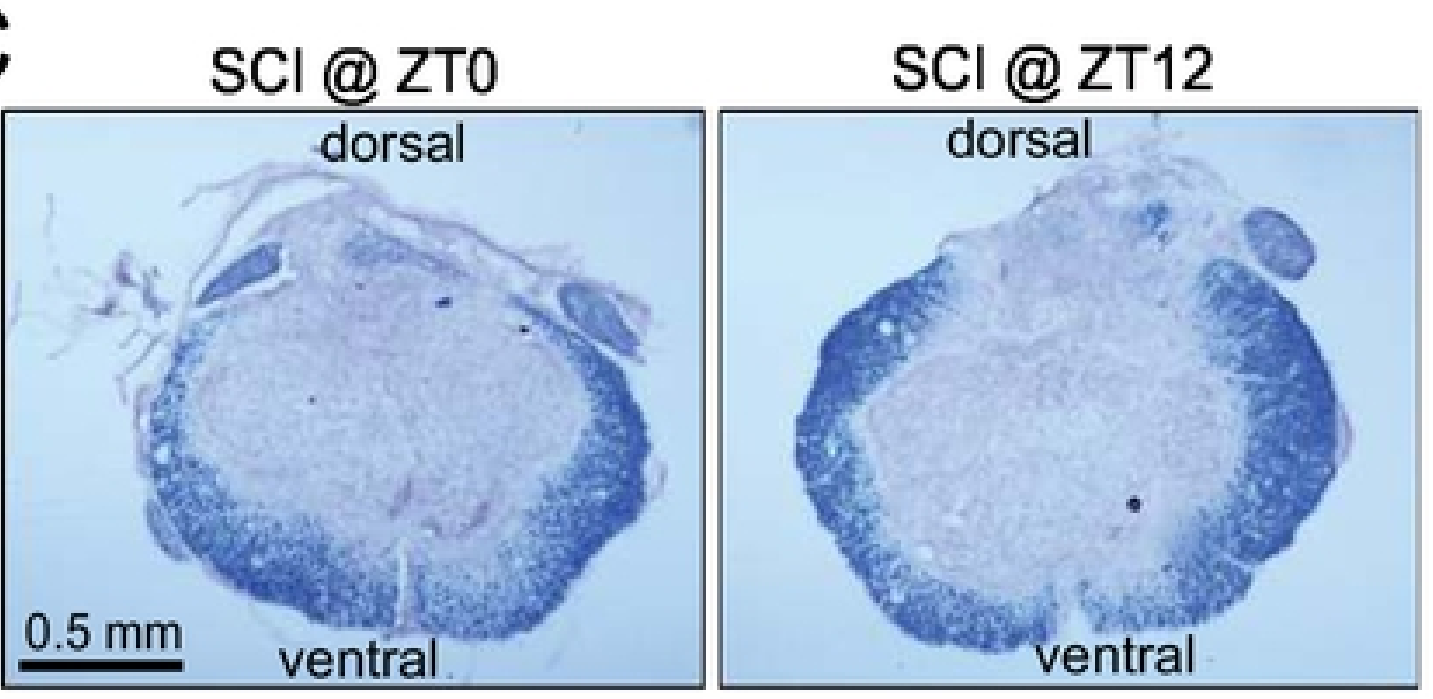

D

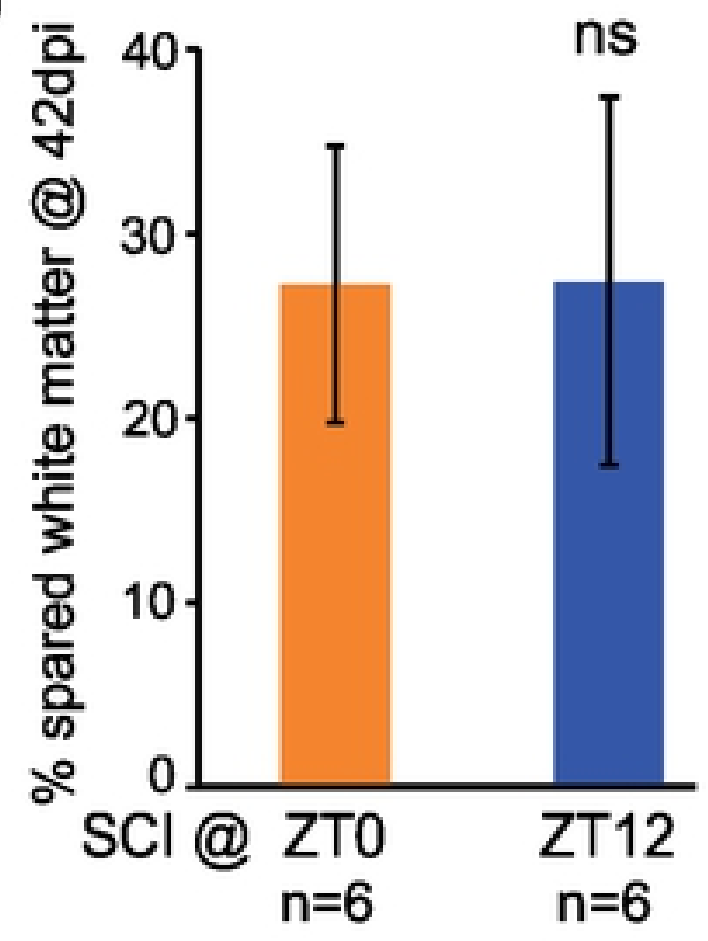

Fig. 2 